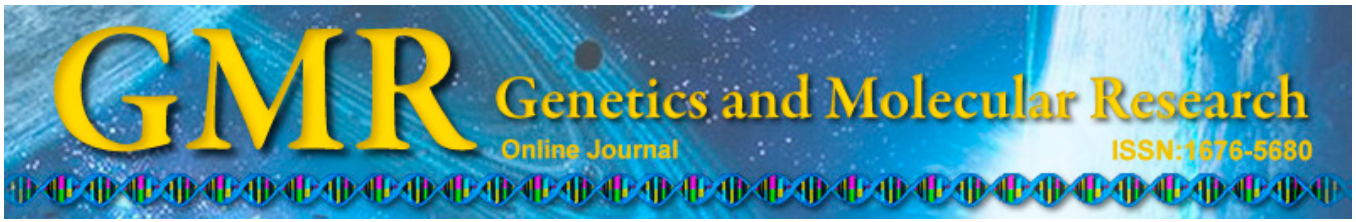

\title{
Serum YKL-40 levels are associated with type 2 diabetes mellitus in patients with obstructive sleep apnea syndrome
}

\author{
L. Sun ${ }^{1}$, J.Y. Liü ${ }^{2}$ and L.R. $\mathrm{Li}^{2}$ \\ ${ }^{1}$ Department of Emergency, Linyi People's Hospital, Linyi, Shandong, China \\ ${ }^{2}$ Department of Pre-Hospital Emergency, Linyi People's Hospital, Linyi, \\ Shandong, China \\ Corresponding author: L.R. Li \\ E-mail: 1ylilr@126.com
}

Genet. Mol. Res. 14 (3): 8919-8925 (2015)

Received January 7, 2015

Accepted April 24, 2015

Published August 3, 2015

DOI http://dx.doi.org/10.4238/2015.August.3.15

\begin{abstract}
Patients with obstructive sleep apnea syndrome (OSAS) have an increased risk for type 2 diabetes mellitus (T2DM). It has been recently demonstrated that serum YKL-40 levels, also named as human cartilage glycoprotein 39, are elevated in T2DM patients. The aim of this study was to determine whether serum YKL-40 levels are associated with T2DM in patients with OSAS. This study consisted of 432 patients with OSAS (234 and 198 patients with and without T2DM, respectively). Serum YKL-40 levels were examined using the enzymelinked immunosorbent assay method. OSAS patients with T2DM had significantly elevated serum YKL-40 levels compared to those without T2DM [205.02 (146.16-272.70) vs 135.72 (114.06-163.38)]. According to the multivariable logistic regression analysis, serum YKL-40 levels were an independent determinant of T2DM in patients with OSAS. Furthermore, based on the linear regression analysis, serum YKL-40 levels were positively associated with the body mass index, systolic blood pressure, serum triglyceride levels, homeostasis model assessment of insulin resistance, and levels of C-reactive protein, fasting plasma
\end{abstract}


glucose, 2-h postprandial plasma glucose, and HbA1c in patients with OSAS. Elevated serum YKL-40 levels may serve as a new biomarker to predict T2DM in patients with OSAS.

Key words: YKL-40; Inflammation; Obstructive sleep apnea syndrome; Type 2 diabetes mellitus

\section{INTRODUCTION}

Obstructive sleep apnea syndrome (OSAS) is characterized by repetitive collapse of the upper airway during sleep, obstruction of airflow, and oxygen desaturation, and could cause sleep arousal (Guven et al., 2012). It is estimated that this disease affects about $4 \%$ of adults (Young et al., 1993). The changes in sleep architecture of OSAS patients contribute to the prominent symptom of daytime sleepiness, which leads to impairments in the quality of life, cognitive performance, and social functioning (Perciaccante et al., 2008). It has been recently demonstrated that OSAS patients have an increased risk of developing glucose intolerance, insulin resistance, and type 2 diabetes mellitus (T2DM) (Rasche et al., 2010).

YKL-40, also referred to as cartilage glycoprotein-39 or chitinase-3-like-1, is a 40$\mathrm{kDa}$ plasma protein without chitinase activity and a member of the mammalian chitinase-like proteins (Rehli et al., 1997). YKL-40 is secreted by activated macrophages and macrophages during the late stage of differentiation, neutrophils, chondrocytes, vascular smooth muscle cells, and cancer cells (Bilim et al., 2010). Although the underlying mechanisms of OSAS are currently not understood, current evidence suggests that inflammation plays a pivotal role in the pathogenesis of OSAS (Carpagnano et al., 2010). A variety of circulating inflammatory biomarkers, such as C-reactive protein (CRP), interleukin-6, and tumor necrosis factor- $\alpha$, were found to be increased in OSAS patients (Aihara et al., 2013). Therefore, we hypothesized that YKL-40 may be involved in the pathogenesis of OSAS. In addition, YKL-40 has been shown to be associated with T2DM. Several investigators have demonstrated that serum YKL-40 levels were elevated in T2DM patients and independently correlated with insulin resistance (Rathcke et al., 2006; Nielsen et al., 2008). These observations indicate a possible association of serum YKL-40 with T2DM in patients with OSAS.

The aim of this study was to determine whether serum YKL-40 levels are associated with T2DM in patients with OSAS.

\section{MATERIAL AND METHODS}

\section{Study population}

This study consisted of 432 patients with OSAS who were referred to the same sleep center of Linyi People's Hospital for the evaluation of suspected OSAS. All participants underwent full-night polysomnography using the Compumedics Voyager Digital Imaging Eseries system (Compumedics Sleep, Melbourne, Australia). OSAS was diagnosed according to an apnea-hypopnea index (AHI) $>5$. Patients with oropharyngeal and dental infections, ongoing dental treatments, periodontal and gingival inflammation, laryngeal pathologies, a history of asthma, chronic bronchitis, allergic rhinitis, chronic obstructive pulmonary disease, and acute airway infection were excluded from the study. T2DM was diagnosed according to 
the following criteria: fasting glucose level $\geq 7.0 \mathrm{mM}$ or 2-h postprandial plasma glucose level $\geq 11.1 \mathrm{mM}$. Patients who had a clinical history of T2DM and were receiving oral hypoglycemic or parental insulin medications were also eligible for this study.

The study was conducted according to the ethics guidelines of the Helsinki Declaration and was approved by the Institutional Research Ethics Board of our hospital. All patients provided written informed consent for participating in this study.

\section{Measurements}

Venous blood samples were obtained during the morning (7-8 am) after an overnight fasting. These samples were separated by centrifugation and stored at $-80^{\circ} \mathrm{C}$. Serum YKL-40 levels were tested using an enzyme-linked immunosorbent assay (Quidel, San Diego, CA, USA). The body mass index (BMI) was calculated as weight in kilograms divided by height squared in meters $\left(\mathrm{kg} / \mathrm{m}^{2}\right)$.

\section{Statistical analysis}

Data are reported as means $\pm \mathrm{SD}$ or medians (interquartile ranges). Statistical analyses were performed using the SPSS version 16.0 software (SPSS, Inc., Chicago, IL, USA). Data normality was analyzed using the Kolmogorov-Smirnov test. Biochemical variables were compared between OSAS patients with and without T2DM using the unpaired $t$-test, MannWhitney U-test, and Chi-square tests, where appropriate. Univariate analysis was performed and variables with a $\mathrm{P}<0.10$ were then entered into a backward stepwise multivariate logistic regression model to assess independent predictors of T2DM. Spearman correlation analysis was performed to evaluate the correlation between parameters. A P value $<0.05$ was considered to be statistically significant.

\section{RESULTS}

\section{Baseline clinical characteristics}

The clinical and laboratory characteristics of OSAS patients with and without T2DM are presented in Table 1. OSAS patients with T2DM showed higher CRP levels than those without T2DM. There were no significant differences in other characteristics between the two groups.

\section{Association of serum YKL-40 levels with T2DM in OSAS patients}

According to the results, serum YKL-40 levels were significantly elevated in OSAS patients with T2DM compared to those without T2DM (Table 1). In the simple logistic regression analysis, high-density lipoprotein $(\mathrm{OR}=0.572,95 \% \mathrm{CI}=0.315-1.038 ; \mathrm{P}=0.066), \mathrm{CRP}(\mathrm{OR}$ $=1.080,95 \% \mathrm{CI}=0.991-1.176 ; \mathrm{P}=0.078)$, and $\mathrm{YKL}-40$ levels $(\mathrm{OR}=1.022,95 \% \mathrm{CI}=1.017$ 1.026; $\mathrm{P}<0.001$ ) showed significant correlations with T2DM (Table 2). Therefore, all of these variables were then entered into a backward stepwise multivariate logistic regression model. According to the multivariate logistic regression, the YKL-40 level remained a significant and independent predictor of T2DM $(\mathrm{OR}=1.023,95 \% \mathrm{CI}=1.018-1.028 ; \mathrm{P}<0.001$; Table 2$)$. 
Table 1. Clinical and biochemical characteristics of obstructive sleep apnea syndrome patients with and without type 2 diabetes mellitus.

\begin{tabular}{lccc}
\hline & OSAS patients without T2DM & OSAS patients with T2DM & P \\
\hline $\mathrm{N}$ & 198 & 234 & \\
Age (years) & $54.48 \pm 12.86$ & $25.84 \pm 10.23$ & 0.745 \\
BMI (kg/m $)$ & $25.11 \pm 3.65$ & $136.41 \pm 19.08$ & 0.123 \\
SBP $(\mathrm{mmHg})$ & $134.19 \pm 22.67$ & $84.62 \pm 11.08$ & 0.270 \\
DBP $(\mathrm{mmHg})$ & $83.97 \pm 13.04$ & $5.28 \pm 1.20$ & 0.578 \\
TC $(\mathrm{mM})$ & $5.22 \pm 1.19$ & $1.91 \pm 0.56$ & 0.623 \\
TG $(\mathrm{mM})$ & $1.94 \pm 0.58$ & $3.52 \pm 1.09$ & 0.841 \\
LDL-C $(\mathrm{mM})$ & $3.39 \pm 1.02$ & $1.26 \pm 0.29$ & 0.286 \\
HDL-C (mM) & $1.32 \pm 0.35$ & $3.89 \pm 0.96$ & 0.065 \\
HOMA-IR & $3.54 \pm 0.91$ & $2.60(1.68-4.60)$ & 0.115 \\
CRP $(\mathrm{mg} / \mathrm{L})$ & $2.20(1.20-3.50)$ & $205.02(146.16-272.70)$ & 0.017 \\
YKL-40 $(\mathrm{ng} / \mathrm{mL})$ & $135.72(114.06-163.38)$ & $<0.001$ \\
\hline
\end{tabular}

The characteristics of CRP and YKL-40 are reported as median (interquartile range); other data are reported as means $\pm \mathrm{SD}$. $\mathrm{BMI}=$ body mass index; $\mathrm{SBP}=$ systolic blood pressure; diastolic blood pressure; $\mathrm{TC}=$ total cholesterol; TG = triglycerides; LDL-C = low-density lipoprotein cholesterol; HDL-C = high-density lipoprotein cholesterol; HOMA-IR = homeostasis model assessment of insulin resistance; $\mathrm{CRP}=\mathrm{C}$-reactive protein; T2DM = type 2 diabetes mellitus; OSAS = obstructive sleep apnea syndrome.

Table 2. Logistic regression analysis for type 2 diabetes mellitus in patients with obstructive sleep apnea syndrome.

\begin{tabular}{|c|c|c|c|c|}
\hline & Simple regression OR $(95 \% \mathrm{CI})$ & $P$ & Multiple regression OR $(95 \% \mathrm{CI})$ & $\mathrm{P}$ \\
\hline Age (years) & $1.003(0.986-1.019)$ & 0.744 & & \\
\hline BMI $\left(\mathrm{kg} / \mathrm{m}^{2}\right)$ & $1.041(0.989-1.095)$ & 0.124 & & \\
\hline $\mathrm{SBP}(\mathrm{mmHg})$ & $1.005(0.996-1.014)$ & 0.270 & & \\
\hline $\mathrm{DBP}(\mathrm{mmHg})$ & $1.005(0.989-1.021)$ & 0.577 & & \\
\hline $\mathrm{TC}(\mathrm{mM})$ & $1.041(0.888-1.221)$ & 0.622 & & \\
\hline $\mathrm{TG}(\mathrm{mM})$ & $0.988(0.877-1.113)$ & 0.841 & & \\
\hline LDL-C (mM) & $1.092(0.926-1.287)$ & 0.296 & & \\
\hline HDL-C (mM) & $0.572(0.315-1.038)$ & 0.066 & $0.575(0.285-1.158)$ & 0.121 \\
\hline HOMA-IR & $1.072(0.983-1.169)$ & 0.118 & & \\
\hline $\mathrm{CRP}(\mathrm{mg} / \mathrm{L})$ & $1.080(0.991-1.176)$ & 0.078 & $1.012(1.003-1.019)$ & 0.008 \\
\hline YKL-40 (ng/mL) & $1.022(1.017-1.026)$ & $<0.001$ & $1.023(1.018-1.028)$ & $<0.001$ \\
\hline
\end{tabular}

$\mathrm{BMI}=$ body mass index; $\mathrm{SBP}=$ systolic blood pressure; diastolic blood pressure; $\mathrm{TC}=$ total cholesterol; $\mathrm{TG}$ = triglycerides; LDL-C = low-density lipoprotein cholesterol; HDL-C = high-density lipoprotein cholesterol; HOMA-IR = homeostasis model assessment of insulin resistance; $\mathrm{CRP}=\mathrm{C}$-reactive protein; $\mathrm{OR}=$ odds ratio; $\mathrm{CI}=$ confidence interval.

\section{Association of serum YKL-40 level with other clinical characteristics}

According to the Spearman correlation analyses, the serum YKL-40 level in OSAS patients was positively correlated with BMI, systolic blood pressure (SBP), serum triglycerides (TG) level, homeostasis model assessment of insulin resistance (HOMA-IR), and CRP level (Table 3).

\section{DISCUSSION}

There were two novel findings in this presents study. Firstly, we determined that OSAS patients with T2DM had higher serum YKL-40 levels than those without T2DM. Elevated YKL-40 levels were significantly associated with T2DM in patients with OSAS. Secondly, serum YKL-40 levels in OSAS subjects were positively correlated with BMI, SBP, TG, 
Table 3. Spearman correlation analyses between YKL-40 and other clinical parameters.

\begin{tabular}{lcr}
\hline Parameters & $\mathrm{r}$ & $\mathrm{P}$ \\
\hline Age $($ years $)$ & 0.026 & 0.597 \\
BMI $\left(\mathrm{kg} / \mathrm{m}^{2}\right)$ & 0.184 & $<0.001$ \\
SBP $(\mathrm{mmHg})$ & 0.168 & $<0.001$ \\
DBP $(\mathrm{mmHg})$ & 0.094 & 0.052 \\
TC $(\mathrm{mM})$ & 0.075 & 0.119 \\
TG $(\mathrm{mM})$ & 0.108 & 0.025 \\
LDL-C $(\mathrm{mM})$ & 0.071 & 0.141 \\
HDL-C (mM) & 0.060 & 0.214 \\
HOMA-IR & 0.224 & $<0.001$ \\
CRP $(\mathrm{mg} / \mathrm{L})$ & 0.413 & $<0.001$ \\
\hline BMI = body mass index; SBP = systolic blood pressure; diastolic blood pressure; TC = total cholesterol; TG \\
= triglycerides; LDL-C = low-density lipoprotein cholesterol; HDL-C = high-density lipoprotein cholesterol; \\
HOMA-IR = homeostasis model assessment of insulin resistance; CRP = C-reactive protein.
\end{tabular}

HOMA-IR, and CRP. To the best of our knowledge, this was the first cross-sectional study in which an association between serum YKL-40 levels and T2DM in patients with OSAS has been demonstrated.

It has been previously reported that OSAS patients had an increased risk of developing impaired glucose tolerance, insulin resistance, and T2DM (Botros et al., 2009). According to the Wisconsin Sleep Study, a total of $14.7 \%$ of subjects with an AHI $>15$ (moderate and severe OSAS) had a diagnosis of diabetes compared to $2.8 \%$ of subjects with an AHI $<5$ (no OSAS), which indicates a greater prevalence of diabetes in patients with OSAS (Reichmuth et al., 2005). Furthermore, there is a high rate of mortality and morbidity among patients who have both T2DM and OSAS (Reutens, 2013). Therefore, it is important to assess the risk of developing T2DM in OSAS patients. We showed that OSAS patients with T2DM had higher serum YKL-40 levels than those without T2DM. Serum YKL-40 levels may serve as a risk predictive biomarker for T2DM in OSAS patients. Other investigators have also found an association between serum YKL-40 levels and T2DM. Circulating concentrations and visceral adipose tissue expressions of YKL-40 were significantly higher in obese patients with T2DM compared to both lean and obese normoglycemic volunteers. In addition, circulating concentrations and visceral adipose tissue expressions of YKL-40 were associated with variables of insulin resistance (Catalán et al., 2011). In another study, serum YKL-40 levels predicted the mortality of patients with T2DM (Persson et al., 2012). These results suggest that YKL-40 may be involved in the pathogenesis of T2DM, especially in OSAS patients.

YKL-40 was found to act as an inflammatory marker in both acute and chronic inflammation. Serum YKL-40 levels were correlated with CRP levels in patients with acute ST-segment elevation myocardial infarction and stable coronary artery disease (Wang et al., 2008). In addition, serum YKL-40 levels were found to be significantly associated with CRP in patients with early rheumatoid arthritis (Harvey et al., 2000). Similar results were found in our study, which indicated an association of serum YKL-40 levels with CRP. Inflammation plays an important role in both OSAS and T2DM. Therefore, these results indicate a possible inflammatory effect of YKL-40 in the mechanism of T2DM development in OSAS patients.

According to the current results, serum YKL-40 levels were correlated with BMI, SBP, HOMA-IR, and TG, which are multiple components of metabolic syndrome. Similarly, other investigators have demonstrated that high serum YKL-40 levels were significantly related to 
some key markers of metabolic syndrome in patients with T2DM and polycystic ovary syndrome (Rathcke et al., 2006; Røndbjerg et al., 2011; Celik et al., 2012). These findings suggest that YKL-40 may play an important role in the pathogenesis of metabolic syndrome. Furthermore, YKL-40 may be involved in the crosstalk between metabolic syndrome, OSAS, and T2DM.

It should be mentioned that the current study inevitably has some limitations. Firstly, the modest sample size in this study could impede our ability to determine weak associations. A larger sample size is required to determine whether the insignificant results were attributed to false negative data. Secondly, this was designed as a cross-sectional study. A prospective cohort study may provide a more definitive evidence for the increase of YKL-40 levels due to OSAS. Lastly, we did not determine serum YKL-40 levels in healthy controls. Therefore, we were unable to determine if there was a significant difference in serum YKL-40 levels between healthy controls and OSAS patients without T2DM.

In brief, OSAS patients with T2DM had significantly elevated serum YKL-40 levels compared to those without T2DM. Serum YKL-40 levels were positively correlated with multiple components of metabolic syndrome. Serum YKL-40 levels may be an independent predictor of T2DM in OSAS patients. More longitudinal studies are required to gain insight into the potential utility of YKL-40 as a biochemical determinant of disease progression and prognosis of T2DM in OSAS patients.

\section{Conflicts of interest}

The authors declare no conflict of interest.

\section{REFERENCES}

Aihara K, Oga T, Chihara Y, Harada Y, et al. (2013). Analysis of systemic and airway inflammation in obstructive sleep apnea. Sleep Breath 17: 597-604.

Bilim O, Takeishi Y, Kitahara T, Ishino M, et al. (2010). Serum YKL-40 predicts adverse clinical outcomes in patients with chronic heart failure. J. Card. Fail. 16: 873-879.

Botros N, Concato J, Mohsenin V, Selim B, et al. (2009). Obstructive sleep apnea as a risk factor for type 2 diabetes. Am. J. Med. 122: 1122-1127.

Carpagnano GE, Spanevello A, Sabato R, Depalo A, et al. (2010). Systemic and airway inflammation in sleep apnea and obesity: the role of ICAM-1 and IL-8. Transl. Res. 155: 35-43.

Catalán V, Gómez-Ambrosi J, Rodríguez A, Ramírez B, et al. (2011). Increased circulating and visceral adipose tissue expression levels of YKL-40 in obesity-associated type 2 diabetes are related to inflammation: impact of conventional weight loss and gastric bypass. J. Clin. Endocrinol. Metab. 96: 200-209.

Celik C, Abali R, Guzel S, Bastu E, et al. (2012). Elevated circulating levels of YKL-40 are a marker of abnormal glucose tolerance in women with polycystic ovary syndrome. Clin. Endocrinol. 77: 893-897.

Guven SF, Turkkani MH, Ciftci B, Ciftci TU, et al. (2012). The relationship between high-sensitivity C-reactive protein levels and the severity of obstructive sleep apnea. Sleep Breath 16: 217-221.

Harvey S, Whaley J and Eberhardt K (2000). The relationship between serum levels of YKL-40 and disease progression in patients with early rheumatoid arthritis. Scand J. Rheumatol. 29: 391-393.

Nielsen AR, Erikstrup C, Johansen JS, Fischer CP, et al. (2008). Plasma YKL-40: a BMI-independent marker of type 2 diabetes. Diabetes 57: 3078-3082.

Perciaccante A, Fiorentini A, Valente R and Tubani L (2008). Autonomic dysfunction characterizes also subjects with obstructive sleep apnea and normal glucose regulation. Sleep Med. 9: 335; author reply 335-336.

Persson F, Rathcke CN, Gall MA, Parving HH, et al. (2012). High YKL-40 levels predict mortality in patients with type 2 diabetes. Diabetes Res. Clin. Pract. 96: 84-89.

Rasche K, Keller T, Tautz B, Hader C, et al. (2010). Obstructive sleep apnea and type 2 diabetes. Eur. J. Med. Res. 15: 152-156. 
Rathcke CN, Johansen JS and Vestergaard H (2006). YKL-40, a biomarker of inflammation, is elevated in patients with type 2 diabetes and is related to insulin resistance. Inflamm. Res. 55: 53-59.

Rehli M, Krause SW and Andreesen R (1997). Molecular characterization of the gene for human cartilage gp-39 (CHI3L1), a member of the chitinase protein family and marker for late stages of macrophage differentiation. Genomics 43: 221-225.

Reichmuth KJ, Austin D, Skatrud JB and Young T (2005). Association of sleep apnea and type II diabetes: a populationbased study. Am. J. Respir. Crit. Care Med. 172: 1590-1595.

Reutens AT (2013). Epidemiology of diabetic kidney disease. Med. Clin. North Am. 97:1-18.

Røndbjerg AK, Omerovic E, Vestergaard H. (2011). YKL-40 levels are independently associated with albuminuria in type 2 diabetes. Cardiovasc. Diabetol. 10: 54.

Wang Y, Ripa RS, Johansen JS, Gabrielsen A, et al. (2008). YKL-40 a new biomarker in patients with acute coronary syndrome or stable coronary artery disease. Scand. Cardiovasc. J. 42: 295-302.

Young T, Palta M, Dempsey J, Skatrud J, et al. (1993). The occurrence of sleep-disordered breathing among middle-aged adults. N. Engl. J. Med. 328: 1230-1235. 